\title{
The Spinning Particles as a Nonlinear Realizations of the Superworldline Reparametrization Invariance
}

\author{
J. Malinsky, :f V.P. Akulov, fi and N. Abdellatif \\ Department of Physics and Technology, \\ Bronx Community College of the City University of New York, \\ Bronx, New York 10453
}

\author{
A. Pashnev \\ Bogoliubov Laboratory of Theoretical Physics, JINR \\ Dubna, 141980, Russia
}

(Dated: November 8, 2018)

\begin{abstract}
The superdiffeomorphisms invariant description of $N$ - extended spinning particle is constructed in the framework of nonlinear realizations approach. The action is universal for all values of $N$ and describes the time evolution of $D+2$ different group elements of the superdiffeomorphisms group of the $(1, N)$ superspace. The form of this action coincides with the one-dimensional version of the gravity action, analogous to Trautman's one.

PACS numbers: 04.65.+e, 02.20.Tw, 11.15.-q
\end{abstract}

\section{INTRODUCTION}

As is well known there exist several equivalent formulations of the massless relativistic particles. The second order and first order formalisms are examples of them. One more example is the conformally invariant description [1], which starts from $D+2$ dimensional spacetime. The existence of alternative approaches always sheds some new light on the nature of the physical system. In particular, as opposite to the conventional description of relativistic massless particles, when the particle coordinates and einbein being the fundamental variables play essentially different roles, in the conformally invariant description both of these types of variables are constructed from the more fundamental ones 1, 2. The geometrical nature of these initial variables has been understood[3]-[6] in the framework of the nonlinear realizations approach and their connection with the dilaton(s) of the reparametrization symmetry of the worldline of the particle was established.

When it applied to different physical systems, the method of nonlinear realizations $\sqrt{7}]-[9]$ allows to understand the geometrical meaning of the basic variables of the system and construct the corresponding Lagrangians following some standard procedure.

As was shown in [10], gravity can be treated as a nonlinear realization of the four dimensional diffeomorphisms group. The consideration was based on the fact that infinite dimensional diffeomorphisms group in four dimensional space can be represented as the closure of two finite dimensional groups - conformal and affine ones [11]. As a consequence of such representation of the diffeomor-

\footnotetext{
*Also at Department of Biomathematics, Box 1213, Mount Sinai Medical Center, New York. New York 10028

$\dagger$ Electronic address: akulov@juno.com

${ }^{\ddagger}$ Electronic address: pashnev@thsun1.jinr.ru
}

phisms group, the basic field in this consideration was the symmetric tensor field of the second rank - the metric field $g_{m, n}$, which corresponds to symmetric generators of the affine group. The generalization of this approach to the case of superspace was given in [12].

Alternatively, one can consider nonlinear realization of the whole infinite dimensional diffeomorphisms group of the arbitrary (super)space. Among the coordinates parameterizing the group element (coset space) in such realization there exist usual coordinates of the (super)space. The vielbeins and connections are represented by the other coordinates of the coset space. This approach in the case of $D$ - dimensional bosonic space-time naturally leads to the Trautman's 13 description of the gravity in terms of vielbeins and connection 14]. In particular case of $D=1$ the same Lagrangian reproduces the conformally invariant description of the massless relativistic particles [4]. Though this approach was not applied to the $D$ - dimensional superspace (to describe the supergravity), its application to the $(1, N)$ superspace with one bosonic and $N$ Grassmann coordinates $\left(\tau, \vartheta^{a}\right),(a=1,2, \cdots N)$ (spinning particles) reveals many features of the bosonic case.

The einbein and its superpartners, as well as spacetime coordinates along with their own superpartners describing the spin of the particle, are connected to some parameters (dilaton and its superpartners) parameterizing the superdiffeomorphisms group of the proper-time superspace $(1, N)$. As it was shown [6], there exist problems in constructing the action in the superspace approach starting from the case $N=3$. On the other hand, as it was claimed, the component approach, developed in the paper $[6]$ for $N=1$, is applicable for an arbitrary value of $N$.

In the present paper we construct the conformally invariant form 11, 2] of the action for $N$ - extended spinning particle [15, 16] in terms of geometrical quantities of the reparametrization group of the proper-time superspace 
$(1, N)$. The action is invariant with respect to the general $(1, N)$ group of superdiffeomorphisms and coincides with the conformally invariant action[1], 2] after some gauge fixing.

\section{NONLINEAR REALIZATION OF THE REPARAMETRIZATIONS IN THE $(1, N)$ SUPERSPACE}

With the help of the coordinate representation of the generators in an auxiliary $(1, N)$ superspace $\left(s, \eta^{a}\right)$

$$
\begin{aligned}
\underbrace{0 \ldots 0}_{P} a^{a_{1}, a_{2}, \ldots, a_{n}} & =i s^{m} \eta^{a_{1}} \eta^{a_{2} \ldots} \eta^{a_{n}} \frac{\partial}{\partial s}, \\
\underbrace{0 \ldots 0}_{P} a^{a_{1}, a_{2}, \ldots, a_{n}} & =i s^{m} \eta^{a_{1}} \eta^{a_{2}} \ldots \eta^{a_{n}} \frac{\partial}{\partial \eta^{a}}, \quad n \leq N .
\end{aligned}
$$

one can calculate the corresponding algebra of diffeomorphisms and forget about this representation. In what follows these generators will be considered as abstract ones. Using them one can write down the group elements of the diffeomorphisms group. Some parameters in the representation of this group elements transform under the left multiplication as the coordinates of the $(1, N)$ superspace. They actually describe the proper-time superspace of the $N$ - extended spinning particles.

To describe the $D$ - dimensional spinning particle in a conformally invariant way we introduce $(D+2)$ group elements in the following form $(\mathcal{I}=0,1, \ldots, D+1)$ :

$$
\begin{aligned}
G_{\mathcal{I}}= & e^{\mathrm{i} \tau P_{0}} e^{\mathrm{i} \vartheta^{a}(\tau) P_{a}} \\
& e^{\mathrm{i} \rho_{a} P_{0}^{a}} e^{\mathrm{i} \mathcal{C}_{a b} P_{0}^{a b}} e^{\mathrm{i} \chi_{a}\left(P_{0}^{0 a}+\mathrm{i} P_{a}^{0}\right)} e^{\mathrm{i} \zeta_{a b}^{c} P_{c}^{a b}} e^{\mathrm{i} 2 l_{a}^{b} P_{b}^{0 a}} e^{\mathrm{i} \kappa^{a} P_{a}^{00}} \\
& e^{\mathrm{i} \Lambda_{a b} P_{0}^{0 a b}} e^{\mathrm{i} \Lambda_{a} P_{0}^{00 a}} e^{\mathrm{i} \Lambda P_{0}^{000}} \cdots \\
& e^{\mathrm{i} \Theta_{\mathcal{I}}^{a} G_{1 / 2}^{a}} e^{\mathrm{i} \Pi_{\mathcal{I}} L_{1}} e^{\mathrm{i} U_{\mathcal{I}} L_{0}} .
\end{aligned}
$$

The third exponent in the second line is chosen to be such in order to simplify the form of resulting differential invariants.

Strictly speaking, the expression (2) describes the parameterizations of the coset space of the diffeomorphisms group over its $S O(N)$ subgroup generated by the rotations in the odd subspace of the proper-time superspace 19. Since the quantities which will be used for the construction of the action are inert with respect to the stability subgroup, one can consistently restrict the consideration to this coset space.

The elements $G_{\mathcal{I}}$ (2) differ from each other only in the last line, which represents the subgroup, generated by dilation $L_{0}=P_{0}^{0}+1 / 2 P_{a}^{a}$, one dimensional conformal boost $L_{1}=P_{0}^{00}+P_{a}^{0 a}$ and $N$ superconformal transformations $G_{1 / 2}^{a}=-i P_{a}^{0}+P_{0}^{0 a}+P_{b}^{a b}$. So, all $(D+2)$ elements have the same parameters in the group space, except the last three ones $\Theta_{\mathcal{I}}^{a}, \Pi_{\mathcal{I}}, U_{\mathcal{I}}$. This property of the elements is unchanged after the left action

$$
G_{\mathcal{I}}^{\prime}=G_{0} G_{\mathcal{I}}
$$

with an arbitrary constant group element $G_{0}$. It is a consequence of the representation

$$
G_{\mathcal{I}}=K H_{\mathcal{I}}
$$

in which parameters of the coset $K$ transform independently from the parameters of the subgroup $H_{\mathcal{I}}[$ [7]- spite of the fact that the element $G_{0}$ is a constant element, it contains infinite number of parameters, similarly to the elements (2). Under such (infinitesimal) transformations

$$
G_{0}=1+i \epsilon
$$

the first two parameters $\tau$ and $\vartheta^{a}$ transform exactly as the coordinates $x^{M}=\left\{\tau, \vartheta^{a}\right\}$ of the $(1, N)$ superspace 14

$$
\delta \tau=\epsilon\left(\tau, \vartheta^{b}\right), \quad \delta \vartheta^{a}=\epsilon^{a}\left(\tau, \vartheta^{b}\right),
$$

where infinitesimal superfunctions $\epsilon\left(\tau, \vartheta^{b}\right)=\epsilon^{0}\left(x^{M}\right)$ and $\epsilon^{a}\left(\tau, \vartheta^{b}\right)=\epsilon^{a}\left(x^{M}\right)$ are constructed out of parameters of the infinitesimal element

$$
\epsilon=\epsilon^{M} P_{M}+\epsilon^{M}{ }_{M_{1}} P^{M_{1}}{ }_{M}+\epsilon^{M}{ }_{M_{1} M_{2}} P^{M_{2} M_{1}}{ }_{M}+\ldots .
$$

which belong to the superdiffeomorphisms algebra. The explicit form of the $\epsilon^{M}(x)$ is the following

$$
\epsilon^{M}(x)=\epsilon^{M}+\epsilon^{M}{ }_{M_{1}} x^{M_{1}}+\epsilon^{M}{ }_{M_{1} M_{2}} x^{M_{2}} x^{M_{1}}+\ldots .
$$

One can consistently consider all other parameters in the group element as superfields - as functions of all these superspace coordinates. As it was already mentioned, such superfield approach faces difficulties when $N \geq 3$. So, in this paper we will consider the so called spontaneously broken realization, i.e., when the parameters $\vartheta^{a}$ instead of being the Grassmann coordinates of the superspace are the Goldstone fields $\vartheta^{a}(\tau)$ which depend on only bosonic coordinate $\tau$. All other parameters are the functions of $\tau$ as well, though we do not write this explicitly for shortness. The transformation laws (6) look in this case as

$$
\delta \tau=\epsilon\left(\tau, \vartheta^{b}(\tau)\right), \quad \delta \vartheta^{a}=\epsilon^{a}\left(\tau, \vartheta^{b}(\tau)\right) .
$$

\section{CONSTRUCTION OF THE INVARIANT ACTION}

As is well known, the Cartan's differential forms $\Omega_{\mathcal{I}}=$ $G_{\mathcal{I}}^{-1} d G_{\mathcal{I}}$ are invariant under the transformations (3). Their expansion coefficients at different generators in the series

$$
\Omega_{\mathcal{I}}=i \omega_{\mathcal{I}}^{0} P_{0}+i \omega_{\mathcal{I}}^{a} P_{a}+\ldots+i \omega_{\mathcal{I}_{00}}^{0} P_{0}^{00}+\ldots
$$

are invariant differential one-forms which can be used for the construction of invariant action integrals. In onedimensional space which is under the consideration the 
external products of one-forms vanish and the only nonvanishing differential invariants are linear combinations of the coefficients $\omega_{\mathcal{I}}$.

Consider the following expression for the action

$$
S=-\frac{1}{2} \Sigma_{\mathcal{I}} \int \omega_{\mathcal{I}_{00}}^{0},
$$

where $\Sigma_{\mathcal{I}}=(-++\ldots++-)$ is the signature of $D+2$ - dimensional space-time and summation over external index $\mathcal{I}$ is assumed.

The action (11) exactly coincides with the action for $N=1$ spinning particle 6 and is analogous to the Trautman's formulation]13] of the gravity (in the form which admits the one-dimensional consideration[14). The expression for the omega-form $\omega_{\mathcal{I}}^{0}$ is rather complicated and contains all parameters explicitly written in the expression (2). To make the connection with the known action for the spinning particle transparent, one have to gauge away some of them. Indeed, the transformation law (6) shows that the Goldstone fields $\vartheta^{a}$ can be vanished by the appropriate choice of the parameters $\epsilon^{a}\left(\tau, \vartheta^{b}\right)$. Moreover, the residual symmetry can be used to exclude some additional fields 14, namely the fields $\rho_{a}, \mathcal{C}_{a b}$ and $\zeta_{a b}^{c}$. Indeed, the transformation laws of all these fields contain terms, proportional to the derivatives of the transformation parameters $\epsilon\left(\tau, \vartheta^{b}(\tau)\right)$ and $\epsilon^{a}\left(\tau, \vartheta^{b}(\tau)\right)[14$ :

$$
\begin{aligned}
\delta \rho_{a}(\tau) & =\frac{\delta}{\delta \vartheta^{a}(\tau)} \epsilon\left(\tau, \vartheta^{d}(\tau)\right)+\cdots, \\
\delta C_{a b}(\tau) & =\frac{\delta^{2}}{\delta \vartheta^{a}(\tau) \delta \vartheta^{b}(\tau)} \epsilon\left(\tau, \vartheta^{d}(\tau)\right)+\cdots, \\
\delta \zeta_{a b}^{c}(\tau) & =\frac{\delta^{2}}{\delta \vartheta^{a}(\tau) \delta \vartheta^{b}(\tau)} \epsilon^{c}\left(\tau, \vartheta^{d}(\tau)\right)+\cdots .
\end{aligned}
$$

These derivatives are indeed the variational derivatives and they have to be calculated at the point $\vartheta^{a}(\tau)=0$ because we already have made this gauge choice.

The explicit expression for the $\omega_{\mathcal{I}_{00}^{0}}^{0}$ in this gauge is

$$
\begin{aligned}
\omega_{\mathcal{I}}^{0}= & d \tau e^{U_{\mathcal{I}}}\left\{\Pi_{\mathcal{I}}^{2}+\dot{\Pi}_{\mathcal{I}}-i \dot{\Theta}_{\mathcal{I}}^{a} \Theta_{\mathcal{I}}^{a}-\right. \\
& -3 \Lambda-\chi_{a} \kappa_{a}+\dot{\chi}_{a} \chi_{a}-i \chi_{a} \Lambda_{a}+ \\
& 2 i \Lambda_{a} \Theta_{\mathcal{I}}^{a}-2 \kappa_{a} \Theta_{\mathcal{I}}^{a}-2 \Lambda_{a b} \chi_{b} \Theta_{\mathcal{I}}^{a}-2 i \mathfrak{A}_{a b} \chi_{b} \Theta_{\mathcal{I}}^{a}+ \\
& \left.\Lambda_{a b} \Theta_{\mathcal{I}}^{a} \Theta_{\mathcal{I}}^{b}-i \mathfrak{A}_{a b} \Theta_{\mathcal{I}}^{a} \Theta_{\mathcal{I}}^{b}\right\} .
\end{aligned}
$$

The rest of parameters $\chi_{a}, \mathfrak{A}_{a}^{b}$ and $\kappa^{a}$ are eated by parameters $\Lambda_{\tilde{\Lambda}} \Lambda_{a}$ and $\Lambda_{a b}$ which become, correspondingly, $\tilde{\Lambda}, \tilde{\Lambda}_{a}$ and $\tilde{\Lambda}_{a b}$ :

$$
\begin{aligned}
\tilde{\Lambda} & =\Lambda+\frac{1}{3} \chi_{a} \kappa_{a}-\frac{1}{3} \dot{\chi}_{a} \chi_{a}+\frac{i}{3} \chi_{a} \Lambda_{a} \\
\tilde{\Lambda}_{a} & =\Lambda_{a}+i \kappa_{a}+i \Lambda_{a b} \chi_{b}-\mathfrak{A}_{a b} \chi_{b} \\
\tilde{\Lambda}_{a b} & =\Lambda_{a b}-i \mathfrak{A}_{a b} .
\end{aligned}
$$

In addition, one can eliminate the field $\Pi_{\mathcal{I}}$ with the help of its equation of motion

$$
\Pi_{\mathcal{I}}=\frac{1}{2} \dot{U}_{\mathcal{I}} .
$$

In terms of new variables

$$
x_{\mathcal{I}}=e^{U_{\mathcal{I}} / 2}, \quad \Psi_{\mathcal{I}}^{a}=e^{U_{\mathcal{I}} / 2} \Theta_{\mathcal{I}}^{a}
$$

the action (11) gets the familiar form 2]

$$
\begin{aligned}
S= & \frac{1}{2} \int \mathrm{d} \tau\left(\dot{x}_{\mathcal{I}}^{2}+i \dot{\Psi}_{\mathcal{I}}^{a} \Psi_{\mathcal{I}}^{a}+\right. \\
& \left.3 \tilde{\Lambda} x_{\mathcal{I}}^{2}-2 i \tilde{\Lambda}_{a} \Psi_{\mathcal{I}}^{a} x_{\mathcal{I}}-i \tilde{\Lambda}_{a b} \Psi_{\mathcal{I}}^{a} \Psi_{\mathcal{I}}^{b}\right) .
\end{aligned}
$$

So, the action (11) which is invariant with respect to the diffeomorphisms group of the proper-time superspace $(1, N)$ properly describes the $N$ - extended spinning particle.

\section{CONCLUSIONS}

In the framework of nonlinear realizations of infinite - dimensional diffeomorphisms groups of the $(1, N)$ superspace we have constructed the reparametrization invariant actions for $N$-extended spinning particle in arbitrary dimension $D$. It is achieved by simultaneous consideration of $D+2$ group elements. The parameters of corresponding group points include the coordinates and momenta of the particle. The interaction between coordinates which effectively reduces the number of the space-time dimensions from $D+2$ to $D$ is included in the action by the presence of the parameters with higher dimensions, which play the role of the Lagrange multipliers and are the same for all considered $D+2$ points on the group space.

It is worth mentioning, that the action obtained for the $N$ - extended spinning particle have the same form as the Trautman's 13 action for the gravity [14. The first of them is invariant under the reparametrizations of the $(1, N)$ superspace, having only one bosonic and $N$ Grassmann coordinates. The second one is written down in $D$-dimensional bosonic space-time. This analogy is very intriguing. So, it would be interesting to apply the method developed here to higher dimensional superspaces to construct corresponding supergravity theories. In particular, such approach in two-dimensional (super)space can be useful to construct the Marnelius like description of the bosonic strings and superstrings. The another possibility is the analogous consideration of the nonlinearly realized $\mathrm{W}$-algebras giving the symmetries of the particle with rigidity.

\section{Acknowledgments}

A.P. would like to thank Prof. S. Catto for the kind hospitality in the City University of New York and Profs. P. Pasti, D. Sorokin and M. Tonin for valuable discussions and for the kind hospitality in the University of Padova, where the essential part of this work was done. The work was supported in part by the INTAS grant 00-00254. 
[1] R. Marnelius, Phys. Rev. D20 (1979) 2091.

[2] W. Siegel, Int. J. Mod. Phys. A3 (1988) 2713.

[3] A. Pashnev, Massless and spinning particles as dynamics in one-dimensional superdiffeomorphism groups, Preprint JINR-E2-99-42 (1999); e-Print Archive: hepth/9902143.

[4] A. Pashnev, Infinite dimensional symmetries and particle models, Proceedings of the XIV-th Max Born Symposium, Karpach, Poland 1999, 143-150

[5] A.I. Pashnev, Czech. J. Phys. 50 (2000) 1335.

[6] A. Pashnev, Nucl.Phys.Proc.Suppl. 102 (2001) 240

[7] S. Coleman, J. Wess and B. Zumino, Phys.Rev., 177 (1969) 2239

[8] C. Callan, S. Coleman, J. Wess and B. Zumino, Phys.Rev., 177 (1969) 2347

[9] D.V. Volkov, Sov.J.Part.Nucl., 43.

[10] A.B. Borisov and V.I. Ogievetsky, Theor.Math.Phys., 21 (1974) 329

[11] V.I. Ogievetsky, Lett.Nuovo Cim., 8 (1973) 988
[12] E.A. Ivanov, I. Niederle, Phys.Rev., bf D45 (1992) 4545

[13] A. Trautman, Bull. Acad. Pol. Sci. 20 (1972) 185; 20 (1972) 503; 20 (1972) 895; 21 (1972) 345.

[14] A. Pashnev, Nonlinear realizations of the (super)diffeomorphism groups, geometrical objects and integral invariants in the superspace, Preprint JINR E2-97-122 (1997); e-Print archive: hep-th/9704203.

[15] V.D. Gershun and V.I. Tkach, JETP Lett. 29 (1979) 320.

[16] P. Howe, S. Penati, M. Pernici and P. Townsend, Phys. Lett. B215 (1988) 555.

[17] E. Ivanov and S. Krivonos, Lett.Math. Phys., 7 (1983) 523 ; ibid 8 (1984) 39

[18] E. Ivanov and S. Krivonos, J.Phys. A: Math.Gen., 17 (1984) L671

[19] The parameterizations like (2) was firstly introduced in [17, 18 for 2-dimensional (super)conformal groups. 